those who were not in 5-year terminal remission and not receiving medication; it was 5\% among subjects with idiopathic or cryptogenic epilepsy, none younger than 14 years of age; $15 \%$ if not in 5-year terminal remission with no medication. Increased risk of death associated with childhood-onset epilepsy and persisting into adulthood is limited to subjects with unremitting seizures and those with another neurologic disability, particularly cognitive impairment. Absence of 5-year terminal remission from epilepsy was the only significant risk factor for sudden, unexplained death. (Sillanpaa M, Shinnar S. Long-term mortality in childhood-onset epilepsy. N Engl J Med Dec 23, 2010;363(26):2522-2529) (Response and reprints: Dr Shinnar, Comprehensive Epilepsy Management Center, Montefiore Medical Center, 111 E $210^{\text {th }} \mathrm{St}$, Bronx, NY 10467. Email: sshinnar@montefiore.org).

COMMENT. SUDEP is usually associated with refractory epilepsy, and AED polytherapy and frequent dose changes, reported as possible causative factors (Tomson $\mathrm{T}$ et al, 2005), are probably a consequence of the recurrent seizures. Seizure-induced cardiac asystole, recorded in patients undergoing video-EEG monitoring, is associated with a risk of SUDEP. (Lanz $\mathrm{M}$ et al, 2010). Also, positional airway obstruction and hypoventilation in postictal coma is suspected in a patient with SUDEP after having a generalized seizure in the prone position. (Tao JX et al, 2010). SUDEP may share a similar etiology with SIDS, and may be preventable in some cases by attention to airway obstruction. Postictal shutdown of brain activity and respiratory arrest is another mechanism proposed. (Lhatoo SD et al, 2010). In cognitively delayed children with remote symptomatic epilepsy, seizures should be kept to a minimum, with avoidance of polytherapy when possible, and observance of seizure precautions related to swimming and sleeping.

\title{
ELECTROCLINICAL CASE-CONTROL STUDY OF SUDEP AND POSTICTAL BRAIN SHUTDOWN
}

Ten adult patients with 30 documented epileptic seizures during video-EEG recording and who later died of SUDEP were compared with 30 matched live controls with 92 epileptic seizures from the same video-telemetry database at Case Medical Center, Cleveland, OH, and Frenchay Hospital, Bristol, UK. Postictal generalized EEG suppression (PGES) was seen in 15/30 (50\%) case and 35/92 (38\%) control seizures. PGES was significantly longer in the generalized motor seizures of the SUDEP group $(\mathrm{P}<0.001)$. Odds of SUDEP occurring were elevated with PGES durations of $>50$ secs $(\mathrm{P}<0.05)$; the odds were quadrupled beyond $80 \mathrm{secs}$. For each $1 \mathrm{sec}$ increase in duration of PGES, the odds of SUDEP increased by $1.7 \%(\mathrm{P}<0.005)$. Refractory epilepsy patients at risk of SUDEP have prolonged PGES ( $>50 \mathrm{secs}$ ), and risk of SUDEP is correlated with duration of PGES. The authors propose that a profound postictal cerebral dysfunction leading to central apnea may be a pathogenic mechanism for SUDEP. (Lhatoo SD, Faulkner HJ, Dembny K, Trippick K, Johnson C, Bird JM. An electroclinical casecontrol study of sudden unexpected death in epilepsy. Ann Neurol Dec 2010;68:787796). (Respond: Dr Lhatoo, University Hospitals, Case Medical Center, Department of Neurology, 11100 Euclid Ave, Cleveland, OH 41106. E-mail: slhatoo@aol.com). 
COMMENT. The prognostic value of video-EEG monitoring and estimation of risk of SUDEP in patients with refractory generalized seizures is demonstrated in this study. Patients at risk should receive more aggressive medical and possibly surgical therapy as well as close postictal observation and stimulation to minimize the occurrence of central inhibition, apnea and SUDEP. Dr LJ Hirsch, Neurological Institute, New York, $\mathrm{NY}$, in an editorial, recommends the use of multimodal ambulatory monitoring (respiration, oxygen saturation, CO2, EKG, and EEG) in epilepsy patients at risk of SUDEP. He cites a prospective, international study in Europe and Australia, using cardiorespiratory and EEG monitoring in 16 cases of SUDEP, reporting central shutdown and respiratory arrest as the primary mechanism of death. (Findings presented at recent European Congress of Epileptology). The quality of life of patients with epilepsy needs protection, but the increasing interest in SUDEP indicates the need for closer monitoring of patients at risk.

\section{RISK FOR DEVELOPING EPILEPSY}

Lifetime risk and cumulative incidence of epilepsy were examined among Rochester, MN, residents between 1960 and 1979, and are reported from Columbia University, New York; and Mayo Clinic. Rochester, MN. Among 412 individuals identified with incident epilepsy, lifetime risk was $1.6 \%$ to age 50 and $3.0 \%$ to age 80 ; cumulative incidence $0.9 \%$ to age $20,1.7 \%$ to age 50 and $3.4 \%$ to age 80 . Lifetime risk through 87 years of age increased over time from $3.5 \%$ in $1960-69$ to $4.2 \%$ in $1970-79$. One in 26 people will develop epilepsy during their lifetime. (Hesdorffer DC, Logroscino G, Benn EKT, Katri N, Cascino G, Hauser WA. Estimating risk for developing epilepsy. A population-based study in Rochester, Minnesota. Neurology January 2011;76:23-27). (Response and reprints: Dr DC Hesdorffer, Columbia University, 630 West $168^{\text {th }}$ St, P\&S Unit 16, New York, NY 10032. E-mail: dch5@ columbia.edu).

COMMENT. Lifetime risk forecasts the need for epilepsy services in a community. The greatest need for these services is in infants and the elderly. The incidence curve varies with age, with high rates in infants under 1 year of age, low rates from age 20 to 60 , and an increase from 60 to age 80 , a time period that is rapidly expanding in the general population. The authors estimate that 12 million individuals in the US will develop epilepsy in their lifetime.

\section{WEST SYNDROME REMISSION FOLLOWING ACUTE VIRAL INFECTION}

Researchers at Nihon University School of Medicine, Tokyo, Japan, report 11 children with intractable epilepsy (West syndrome in 6 and myoclonic seizures in 5) who showed clinical and electrographic improvement following acute viral infection. Seizure remission occurred following exanthem subitum in 5 patients, rotavirus gastroenteritis in 2 , measles in 2 , herpetic stomatitis in 1 , and common cold in 1 . In patients with West syndrome, salaam and/or tonic spasms resolved within 6 days after onset of viral infection, and hypsarrhythmia was modified and evolved to localized spikes. Myoclonic seizures resolved rapidly and completely and the EEG gradually normalized or improved. 